\title{
Spectral sensitivity of the Sooty mangabey
}

CALVIN K. AOAMS ANO ARTHUR E. JOHES

US ARMY MEDICAL RESEARCH LABORATORY. FORT KNOX. KENTUCKY

Purkinje shift and scotopic and photopic spectral sensitivity functions were determined for four Sooty mangabeys and five human controls using a flicker technique in a four-choice discrete trials task. Results indicate a Purkinje shift similar in magnitude and frequency locus to that of human controls. Scotopic spectral sensitivity was almost identical to that of human controls, although both species showed reduced sensitivity relative to the standard CIE function, at short wavelengths. Photopic spectral sensitivity disclosed enhanced sensitivity in the blue and reduced sensitivity (0.3-0.4 log units) in the red relative to human controls.

Several recent reports have indicated that there are marked similarities between the visual functioning of certain infrahurnan primate species and man. These similarities relate not only to normal human trichromatic vision, but also to at least one of the defective forms (protanomaly) as well. It would thus seem that because of these marked similarities, the use of infrahuman primates in the investigation of the mechanisms of human color vision should be particularly fruitful. The present report is one of a planned series of behavioral, physiological, and anatomical studies of the mangabey and other species, with the alm of obtaining a better understanding of human color vision by providing a broader comparative basis on which to interpret visual function.

The mangabey was chosen because this species supposedly has the highest cone density of any primate species reported (Polyak, 1946). This characteristic might well offer some unique advantages. Regardless, these animals are docile compared to Rhesus macaques, so this characteristic alone makes them attractive.

The specific purpose of the present study was to determine, using a flicker technique, if this species has a functionally duplex retina and, if so, the relative spectral sensitivities of the two components. Human controls were run so that direct comparisons to man could be made.

The flicker technique utilized in the present study is based on the result of numerous physchophysical studies with man and has previously been successfully utilized in the testing of monkeys (DeValios, 1965; Jacobs, 1963).

The theory and procedure for determining if the species under test has a duplex retina, and if duplex, the relative sensitivities of each component, is as follows: Using short wavelength light, one determines the Intensity required for off at different flicker frequencies. If the species has a duplex retina similar to man's, the resulting curve will have a distinct "break" (Purkinje shift) in it in the neighborhood of
10-20 cps (Hecht \& Shlaer, 1936). This is supposedly due primarily to two factors: (1) the rods are more sensitive to short wavelength light than the cones, and (2) at low frequencies the rods are primarily the functioning receptors; at high frequencies, the rods can no longer follow, so the cones dominate. When one repeats this procedure using long wavelength light, the resulting curve should be continuous since the two systems have about equal sensitivity in this region of the spectrum. If white light is used, the results should be intermediate between the previous two because all wavelengths are present. Once the presence of a duplex retina has been established. one can then determine the spectral sensitivity of each component by selecting a flicker frequency that falls clearly in the domain of that component and determine eff as a function of intensity across several spectral loci.

\section{Subjects}

\section{METHOD}

Four adolescent Sooty mangabeys (Cercocebus lorquatys atys) with no previous test experience served as Ss. Three were male, one female. The human control Ss were five young adult men with normal color vision (as determined by the Farnsworth-Munsell 100-Hue test).

\section{Apparatus}

The testing situation was a four choice discrimination in which $S$ had to discriminate one flickering stimulus from three steady ones under dark adapted conditions. Four stimulus beams taken from two tungsten ribbon-filament lamps were each passed through separate circular diffusing windows $1-1 / 16$ in. In diameter in the light-tight testing chamber. On each trial one of the beams was interrupted at a point aperture by a movable episcotister which had interchangeable discs, all of which yielded a lightdark ratio of $1: 1$. Correction for Talbot brightness $(0.3 \log \pm 0.2 \log$ units systematically varied across windows over trials) was inserted in the three steady bearns to control for brightness cues. When the monkey pressed the "flickering" window, it received a $97 \mathrm{mg}$ dextrose pellet from the food cup below that window. Between trials all beams were interrupted by a movable beam block. Vertical restraining bars 6 in. behind the four stimulus windows restricted the retinal subtense of each window to a maximum of $10^{\circ}$. All surfaces of the interior of the test chamber were flat black.

The intensity and spectral composition of each of the four beams were controlled by Wratten No. 96 
neutral density filters and Kodak Wratten color filters, respectively. The elght color filters used for the spectral sensitivity functions were Nos. $98,75,45+58$, $74,73,72 \mathrm{~b}, 29$, and 92. Filters No. 45 and 29, plus white light, were used for the Purkinje shift determinations. Wavelength specification of each of the single filters was the dominant wavelength for illuminant A given by the manufacturer. The wavelength specification for the $45+58$ combination was $510 \mathrm{~nm}$, which was the locus of maximum transmission of the combination. Spectral transmission of each of the filters was determined with a Cary Model 11 spectrophotometer. The spectral energy distribution of the tungsten ribbon filaments (operated at $6 \mathrm{~V} 18 \mathrm{~A}$ ) was taken as that of a black body at $3000^{\circ} \mathrm{K}$, the computed color temperature for that voltage and amperage. From these data the density correction required for each filter to produce an equal energy spectrum was determined.

\section{Procedure}

The objective at each wavelength-flicker frequency combination was to determine that intensity of light at which S's ability to discriminate the flickering stimulus from the three steady ones reached the $50 \%$ point.

The method of constant stimuli was used. For each wavelength-flicker frequency combination, five light intensities spaced $0.3 \mathrm{log}$ units apart were selected such that percent correct responses ranged from near perfect to chance (25\%). Five-trial blocks at each of these intensities, in random order, were given on each of four sessions. The data for the four sessions were then averaged and an intensity by percent correct response function drawn. The intensity yielding $50 \%$ correct responses was then determined by interpolation, corrected for energy transmission for that particular filter, and used as the measure of performance for that animal at that wavelength-flicker frequency combination.

Testing was conducted five days each week with one session per day. The session on the first day of each week was considered as a warm-up to stabilize performance; only data from the last four days each week were utilized in tabulations. Each session consisted of either 100 trials (sensitivity functions) or 125 trials (Purkinje shift problems). The sequence in which each spectral locus or frequency was tested was counterbalanced over sessions.

$S$ was allowed to dark adapt a minimum of $10 \mathrm{~min}$ before testing was begun. Trials were run 15-20 sec apart with $S$ allowed a maximum of $10 \mathrm{sec}$ within which to respond (latencies were almost always less than $5 \mathrm{sec}$ ). Location of the positive stimulus was varied according to a predetermined random order, with the restriction that all windows were positive an equal number of times (over all trials) and that each window be positive at least once in each fivetrial block.
The human Ss were run under the same general conditions as the monkeys, except that a verbal response and verbal knowledge of results were given. Purkinje shift was determined using white light, and at spectral loci of $486 \mathrm{~nm}$ and $630 \mathrm{~nm}$ across five flicker frequencies, 10-30 cps. Scotopic sensitivity functions were determined using $10 \mathrm{cps}$ flicker, and photopic sensitivity functions using 25 or 30 cps flicker, at eight spectral loci.

\section{Purkinje Shift}

\section{RESULTS}

The individual functions in Fig. 1 show the results of the three determinations of Purkinje shift for each animal. All show a clear rod-cone break with the 486 $\mathrm{nm}$ filter, indicating a duplex retina. The frequency locus of this shift was between 15 and $20 \mathrm{cps}$ for Animals 2 and 3, and between 20 and $25 \mathrm{cps}$ for Animals 1 and 4. No significance is attached to this frequency difference, as the human controls showed comparable variability in shift frequency (three showed a shift between 15 and 20; two showed a shift between 20 and $25 \mathrm{cps}$ ). Comparable data on macaque and Cebus (DeValois, 1965) show a shift between 20 and $25 \mathrm{cps}$, while for the squirrel monkey, the shift is between 15 and 20 cps (Jacobs, 1963). As expected, the results for the $630 \mathrm{~nm}$ filter show no rod-cone break, and the result for white light are intermediate between those for the two color filters.

\section{Scotopic Function}

The individual scotopic functions are shown in Fig. 2 (10 cps curves) and summarlzed and compared

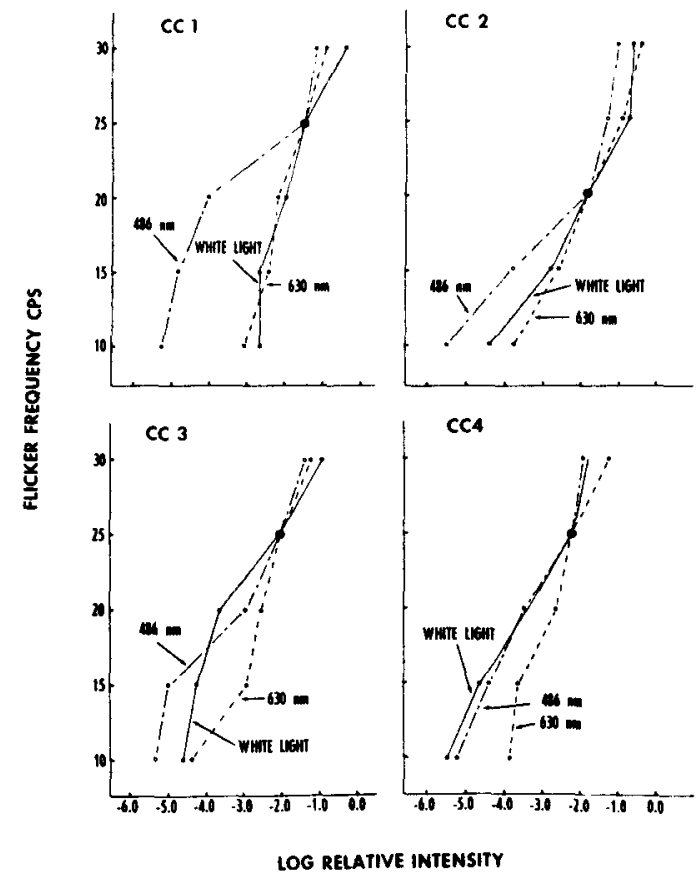

Fig. 1. CFF as a function of $\log$ relative intensity for individual mangabeys under three conditions. Curves are equated at 20 or 25 cps for comparison purposes ( $\mathrm{CC}=$ Cercocebus). 


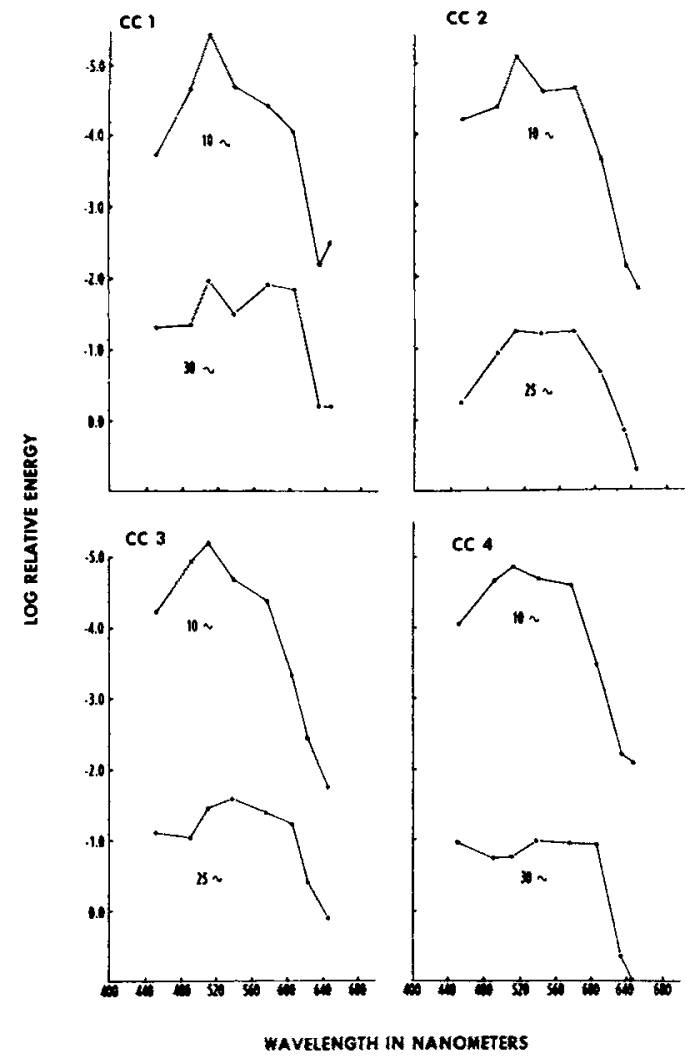

Fig. 2. Individual mangabey scotopic $(10 \sim)$ and photopic $(25$ or $30 \sim$ ) curves. Each curve average of four sessions ( $\mathrm{CC}=$ Cercocebus).

to both the human controls and the CIE curve in Fig. 3. Although showing a somewhat reduced sensitivity in the blue (relative to the CIE function), the curve in Fig. 3 closely parallels that for the human controls, indicating a scotopic sensitivity very similar to that of man.

\section{Photopic Function}

The Individual photoplc functions are also shown in Fig. 2 (25 or $30 \mathrm{cps}$ curves) and summarized and compared to both the human controls and the CIE curve in Fig. 4. In this case the departure from the human data and the CIE function is consistently in the direction of enhanced sensitivity in the blue and slightly reduced sensitivity in the red portion of the spectrum.

\section{DISCUSSION}

The Purkinje shift determinations clearly establish the presence of a duplex retina. The magnitude of the shift, its frequency locus and the energy levels at which the mangabeys perform are very similar to that shown by the human Ss in this experiment, and macaques (DeValois, 1965), and squirrel monkeys (Jacobs, 1963), tested in a very similar apparatus.

The shape of the scotopic function is almost identical to that shown by the human controls, as are the energy levels involved and the degree of individual variabllity. Why both species show a marked reduction in sensitivity (relative to the CIE function) at the blue end of the spectrum is unexplained. It might well be due to some characteristic of the present testing procedure. However, if 1 it is, it applies only to scotopic conditions, as no simllar effect was seen in elther specie in the photopic determinations.

The results for the photopic determinations do not show the same degree of similarity to man as was found in the scotopic determinations. However, the relative energy levels at which performance reached $50 \%$ correct in the middle range of wavelengths, and the degree of individual variability found in each specie was again quite similar. The enhanced sensitivity of the mangabeys in the blue, relative to both the human controls and the CIE function, is essentially that found for other species using this technique (DeValois, 1965; Jacobs, 1963). It is also similar to that recently reported by Schrier and Blough (1966) for the macaque using a much different technique. The reduction in long wavelength sensitivity of the human controls relative to the CIE function was unexpected. There was no indication in the FarnsworthMunsell profiles that any of the control Ss were red weak. The reduced sensitivity of the mangabeys in the long wavelength region relative to the human controls (0.3-0.4 log units) suggests that this species has reduced sensitivity in this region. The magnitude of this reduction closely approximates that reported for Cebus by DeValois (1965).

Because of the many differences in technique, it

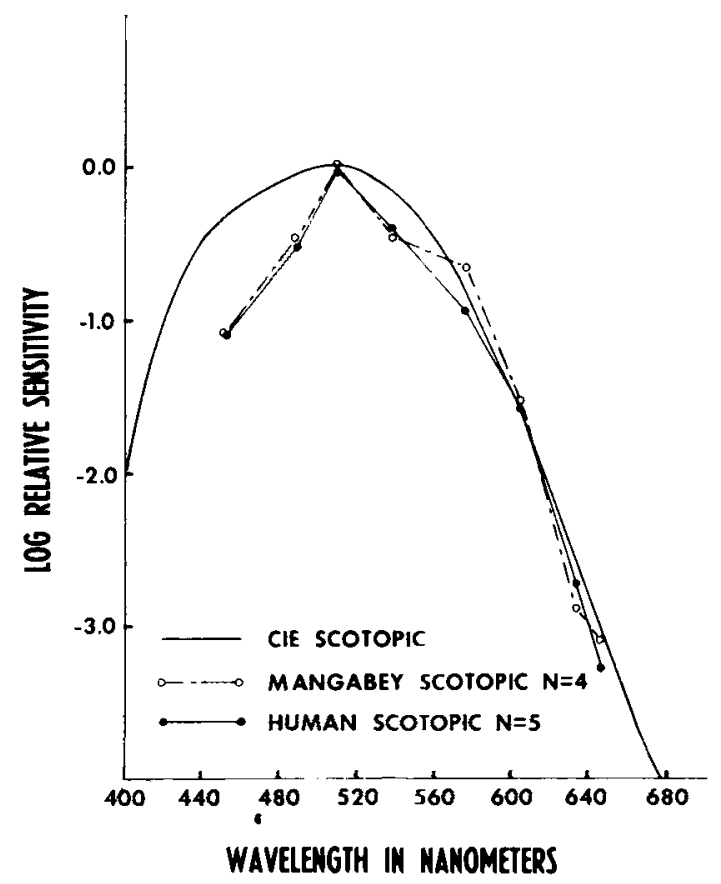

Fig. 3. Average mangabey and human control relative scotopic spectral sensitivity functions. 


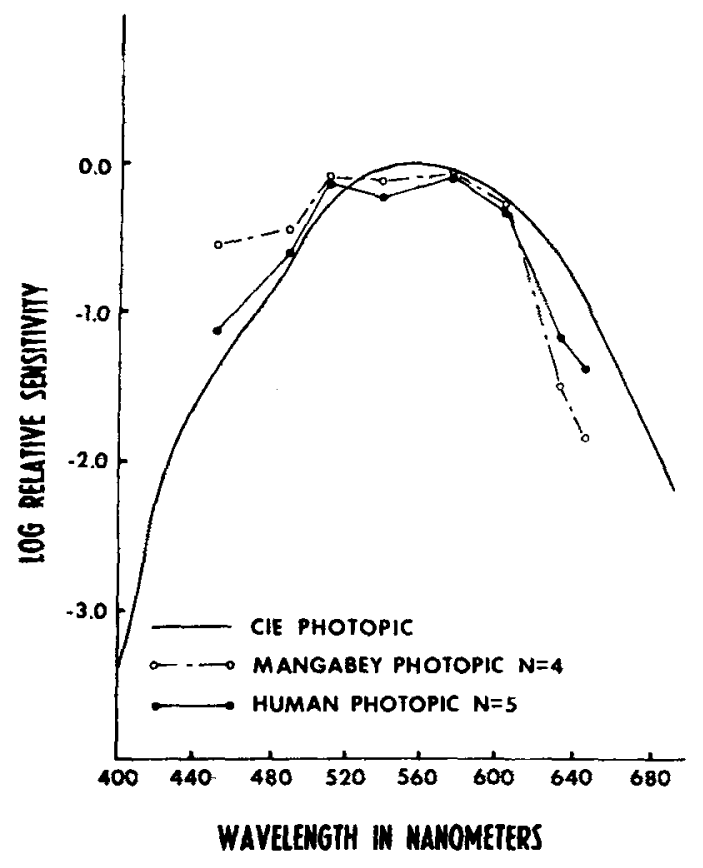

Fig. 4. Average mangabey and human control relative photopic spectral sensitivity functions.

is difficult to compare the present results with those of Sidley, Sperling, Bedarf, and Hiss (1965). However, there are discontinuities in the Individual photopic functions that suggest secondary peaks corresponding to theirs, particularly if one considers the enhancement shown at $452 \mathrm{~nm}$ as a secondary peak. Finally, It should be pointed out that the present results are quite similar to those previously reported for this species by Jones, Polson, and DeValois (1964), using ERG measures.

\section{Reterences}

DeValois, R. L. Behavioral and electrophysiological studies of primate vision. In W. D. Neff (Ed.), Contributions to sensory physiology. New York: Academic Press, 1965. Pp. 137-178.

Hecht, S., \& Shlaer, S. Intermittent stimulation by light. V. The relation between intensity and critical frequency for different parts of the spectrum. J. gen. Physiol., 1936, 19. 965-977.

Jacobs, G. H. Spectral sensitivity and color vision of the squirrel monkey. J. conp. physiol. Psychol., 1963, 56, 616-621.

Jones, A. E., Polson, M. C., \& DeValois, R. L. Mangabey $x$ and $b$ wave electroretinogram components: Their dark-adapted luminosity functions. Science, 1964, 146, 1486-1487.

Polyak, S. L. The retina. Chicago: University of Chicago Press, 1941. P. 256.

Schrier, A. M., \& Blough, D. S. Photopic spectral sensitivity of macaque monkeys, $J$. comp. phy siol. Psychol., 1966, 62, 457-458.

Sidley, N. A., Spering, H. G., Bedar, E. W., \& Hiss, R. H. Photopic spectral sensitivity in the monkey: Methods for determining, and initial results. Science, 1965, 150, 1837-1839.

\section{Notes}

1. Dr. Jones' present address is: Honeywell, Systems and Research Division, 2345 Walnut Street, St. Paul, Minnesota 55113.

2. We wish to acknowledge the assistance of Edward A. Battisti and Thomas D. Werner in the collection and tabulation of the data.

(Accepted for publication May 28, 1967.) 